\title{
Effect of Trifluoromethyl Substitution on C-3 Position in 1H NMR of Quinolones / Coumarins
}

\author{
Anjali Gupta ${ }^{\mathrm{a}, \mathrm{b} *}$, and Rajinder K. Gupta ${ }^{\mathrm{b}}$ \\ ${ }^{a}$ I.T.S. Engineering College, Greater Noida, U.P. \\ ${ }^{b}$ University School of Biotechnology, Guru Gobind Singh Indraprastha University, Sector- \\ 16C, Dwarka, New Delhi-110078, India. \\ AUTHOR INFORMATION \\ Corresponding Author \\ *E-mail: anjali21in@gmail.com; Tel: +91-9211477975.
}

\begin{abstract}
The structural assignment of differently substituted quinolones / coumarins was reviewed using ${ }^{1} \mathrm{H}$ NMR spectral data. In case of quinolones / coumarins, with varied substitutions at C-4 and different positions of the benzenoid ring, there is a great variation in the ${ }^{1} \mathrm{H}$ NMR value of the proton only attached with $\mathrm{C}-3$. It was observed that when different substitutions (methyl / amino / hydroxy / acetoxy / trifluoromethyl) were carried out at C-4 position, maximum deshielding with the proton attached with C-3 was observed with trifluoromethyl substitution. The observation is same even with the variation in different groups at the benzenoid ring.
\end{abstract}

Keywords: NMR; ${ }^{1} \mathrm{H}$; trifluoromethyl; quinolones; coumarins

Academic Discipline And Sub-Disciplines: NMR studies TYPE (METHOD/APPROACH): Analysis of NMR

\section{Council for Innovative Research}

Journal: Journal of Advances in Chemistry

Peer Review Research Publishing System

Vol. 8, No. 3

editor@cirjac.com

www.cirjac.com, member.cirworld.com 


\section{INTRODUCTION}

Quinolones and coumarins are the important heterocyclic system present in biologically active alkaloids (Fig. 1). ${ }^{[1-4]}$ As part of our ongoing research on the synthesis of differently substituted heterocyclic compounds with potential antioxidant and anti-inflammatory activities, ${ }^{[5-8]}$ we were interested in synthesizing the derivatives of quinolones / coumarins containing different substituents on benzenoid ring as well as C-4 position of the heterocyclic ring.

As these moieties are more frequently incorporated in molecules of biological value, special attention has been paid to the effect of various substituents (trifluomethyl / methoxy / hydroxy / amino / acetoxy / nitro / fluoro). In the present work, we have described the effect of substitution at C-4 position as well as benzenoid ring on the $\delta$ value of proton attached with $\mathrm{C}$ 3 position using ${ }^{1} \mathrm{H}$ NMR.<smiles>CCn1c(=O)c(-c2ccccc2)cc2ccc(N(C)C)cc21</smiles><smiles>Cn1c(=O)c(-c2ccccc2)cc2ccc(N)cc21</smiles><smiles>CCn1c(=O)c(-c2cccc(Cl)c2)cc2ccc(N(C)C)cc21</smiles>

Figure 1

\section{RESULTS \& DISCUSSION}

Our route to synthesis of quinolones and coumarins was based on Knorr reaction and Pechmann synthesis respectively between anisidines / phenols and ethyl acetoacetate / ethyl trifluoromethyl acetoacetate (Scheme 1) which were submitted to a sequence of reactions, thereby generating different derivatives of quinolones / coumarins. $^{[8,9]}$

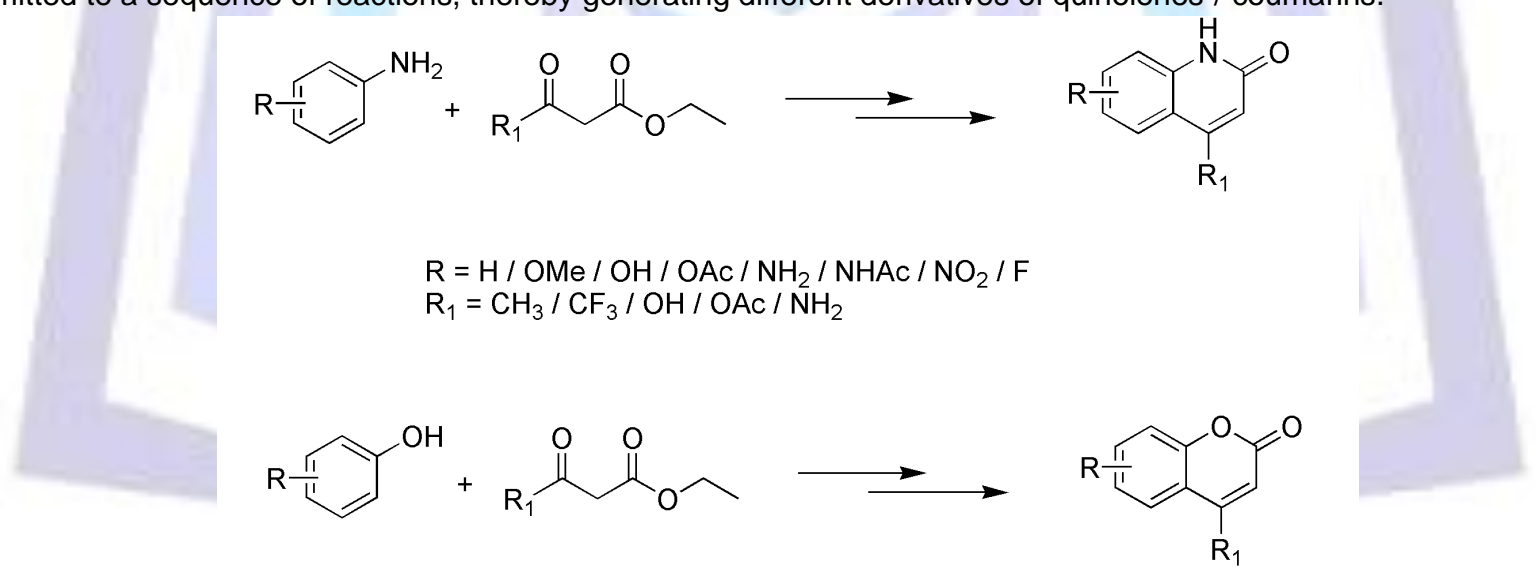

Scheme 1: Schematic route for the synthesis of different quinolone / coumarin analogues.

Application of standard NMR technique to the quinolone / coumarin analogues allowed complete ${ }^{1} \mathrm{H}$ assignment to be made. The effect of various substituents at C-4 position as well as benzenoid ring on the $\delta$ value was found to be significant in case of proton attached with C-3 position rather than other positions.

In case of quinolones bearing different substituents at C-4 position (1-5), the appearing trend is that trifluoromethyl substitution (5) results in maximum deshielding of $\mathrm{H}-3$ position than other substituents at $\mathrm{C}-4$ position (Table 1). It is worth noting that as compared to C-4 methyl group (1), the substitution of hydroxy (3) or amino groups (2) causes shielding effect on $\mathrm{H}-3$. It is also seen that acetylation at $\mathrm{C}-4$ (4) results in deshielding of $\delta$ value. Following these promising observations, the proposed discriminative tool was also checked for other structurally related quinolone / coumarin analogues where variation was carried out on the benzenoid ring.

In case of C-4 methyl quinolone / coumarin derivatives bearing methoxy (6a, 11a and 19a), hydroxy / amino (7a, 9a, 12a, $17 a$ and 19b), acetoxy / urethane groups (8a, 10a, 13a, 16a, 18a and 19c), fluoro (14a) and nitro (15a) groups on the benzenoid ring, deshielding was noticed for the proton attached with $\mathrm{C}-3$, in case of either acetoxy or fluoro substituent on the benzenoid ring but to a very lesser extent (Table 2). It has been observed that the replacement of methoxy group by 
hydroxy group on the benzenoid ring causes upfield effect, whereas opposite effect was observed on the incorporation of acetoxy group in place of hydoxy group and this deshielding due to acetoxy group is even more than methoxy group.

Studies were extended with a view to examine the influence of trifluoromethyl group at C-4 position in place of methyl group. It was observed that the substitution of $\mathrm{C}-4$ methyl group with trifluoromethyl group has a great influence on the ${ }^{1} \mathrm{H}$ NMR shift of the H-3 proton. To illustrate this substitution dependence, one can just compare the ${ }^{1} \mathrm{H}$ NMR data of $\mathrm{C}-4$ methyl analogues with those of $\mathrm{C}-4$ trifluoromethyl derivatives having different substitutions on benzenoid ring. The structural assignments for compounds are reported in Table 2.

In the present report, it is clearly evident that when $\mathrm{C}-4$ methyl group gets replaced with trifluoromethyl, the $\delta$ value for $\mathrm{H}-3$ becomes downfield. It was also observed that in compound 13b, the $\delta$ value for $\mathrm{H}-3$ was found to be highly deshielded i.e., $8.01 \mathrm{ppm} ; \Delta \delta$ being $1.55 \mathrm{ppm}$ which is quite significant. The deshielding effect is also noteworthy in case of nitro substituted quinolone, $15 \mathbf{a}-\mathbf{b}(\Delta \delta=1.06)$ rather than fluoro, $14 \mathrm{a}-\mathrm{b}(\Delta \delta=0.63)$ or amino, 9a-b $(\Delta \delta=0.38), 17 \mathbf{a}-\mathbf{b}(\Delta \delta=$ 0.48 ) analogues.

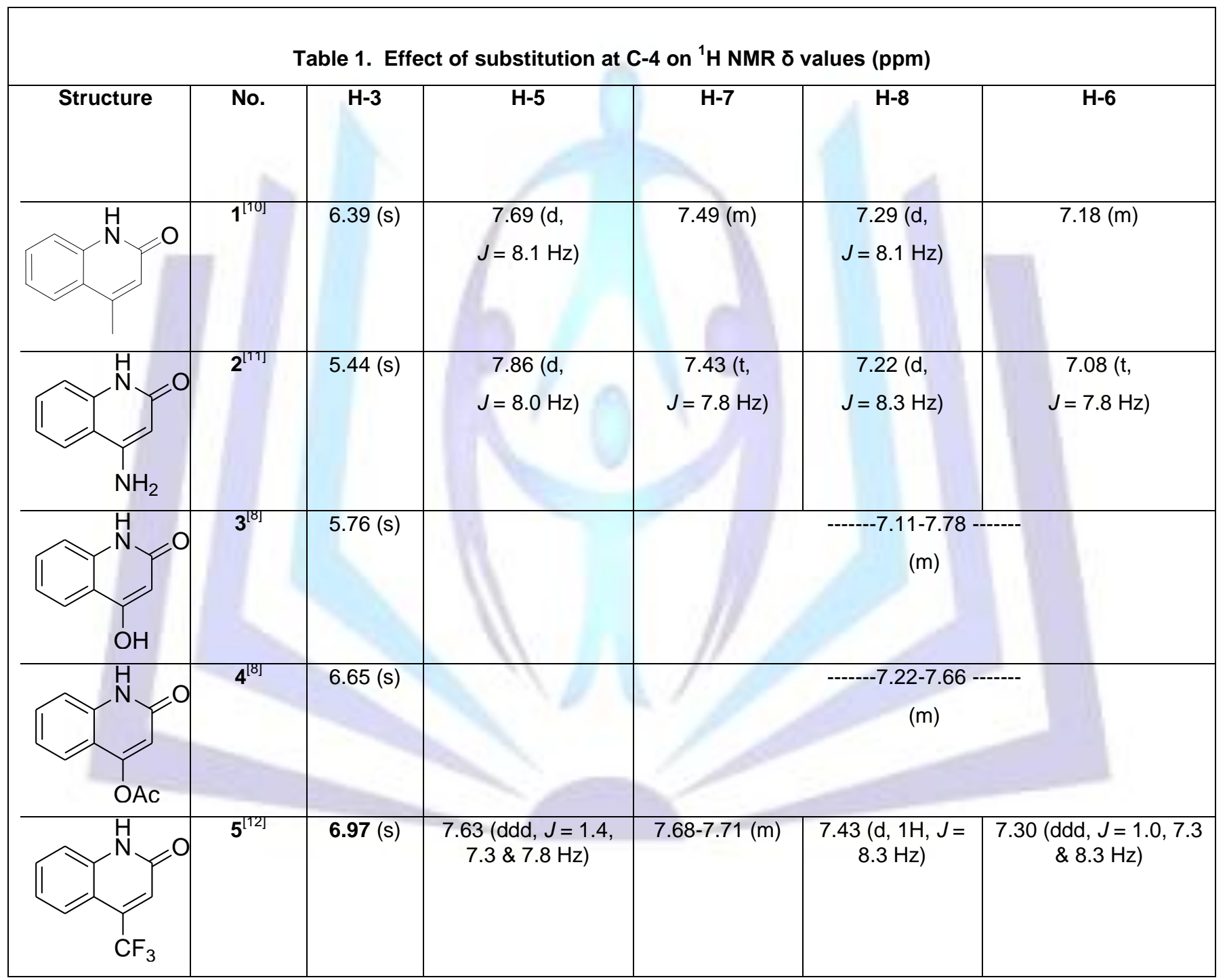


Table 2. ' $\mathrm{H}$ NMR $\delta$ values (ppm) of different quinolone / coumarin derivatives

\begin{tabular}{|c|c|c|c|c|c|}
\hline Structure & Compound & $\mathrm{H}-3$ & $\mathrm{H}-5$ & H-6 / H-7 & $\mathrm{H}-8$ \\
\hline \multirow{2}{*}{ 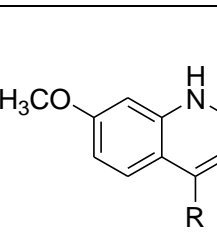 } & $6 \mathbf{a}^{[8]} \mathrm{R}=\mathrm{CH}_{3}$ & $6.30(\mathrm{~s})$ & $\begin{array}{c}7.66(\mathrm{~d}, \\
J=8.9 \mathrm{~Hz})\end{array}$ & $\begin{array}{c}6.84(\mathrm{~d}, \\
J=8.7 \mathrm{~Hz})\end{array}$ & $6.94(\mathrm{~s})$ \\
\hline & $\mathbf{6 b}^{[9]} \mathrm{R}=\mathrm{CF}_{3}$ & $6.77(\mathrm{~s})$ & $\begin{array}{c}7.61(\mathrm{~d}, \\
J=8.4 \mathrm{~Hz})\end{array}$ & \multicolumn{2}{|c|}{--------6.92-6.96--------(m) } \\
\hline \multirow{2}{*}{$\underbrace{H O}_{R}$} & $7 \mathbf{a}^{[8]} \mathrm{R}=\mathrm{CH}_{3}$ & $6.08(\mathrm{~s})$ & $\begin{array}{c}7.45(\mathrm{~d}, \\
J=8.8 \mathrm{~Hz})\end{array}$ & $\begin{array}{c}6.59(\mathrm{~d}, \\
J=8.8 \mathrm{~Hz})\end{array}$ & $\begin{array}{c}6.65(\mathrm{~d}, \\
J=2.4 \mathrm{~Hz})\end{array}$ \\
\hline & $7 \mathbf{b}^{[9]} \mathrm{R}=\mathrm{CF}_{3}$ & $6.68(\mathrm{~s})$ & $----6.76-6.82----(m)$ & $\begin{aligned} & 7.53(\mathrm{dd}, \\
J= & 2.1 \& 8.7 \mathrm{~Hz})\end{aligned}$ & $----6.76-6.82----(m)$ \\
\hline \multirow{2}{*}{$\underbrace{\mathrm{AcO}}_{\mathrm{R}}$} & $8 \mathbf{a}^{[8]} \mathrm{R}=\mathrm{CH}_{3}$ & $6.39(\mathrm{~s})$ & $\begin{array}{c}7.73(\mathrm{~d}, \\
J=6.1 \mathrm{~Hz})\end{array}$ & $\begin{array}{c}6.98(\mathrm{dd}, \\
J=2.4 \& 6.2 \mathrm{~Hz})\end{array}$ & $7.05(\mathrm{~s})$ \\
\hline & $\mathbf{8 b}^{[9]} \mathrm{R}=\mathrm{CF}_{3}$ & $6.97(\mathrm{~s})$ & $\begin{array}{c}7.73(\mathrm{~d}, \\
J=8.7 \mathrm{~Hz})\end{array}$ & $\begin{array}{c}7.12(\mathrm{dd}, \\
J=1.8 \& 8.7 \mathrm{~Hz})\end{array}$ & $\begin{array}{c}7.19(\mathrm{~d}, \\
J=1.8 \mathrm{~Hz})\end{array}$ \\
\hline \multirow{2}{*}{$\mathrm{H}_{\mathrm{R}}^{\mathrm{H}_{2} \mathrm{~N}}$} & $9 a^{[9]} \mathrm{R}=\mathrm{CH}_{3}$ & $5.96(s)$ & $\begin{array}{c}7.34(\mathrm{~d}, \\
J=8.7 \mathrm{~Hz})\end{array}$ & $\begin{array}{c}6.46(\mathrm{~d} \\
J=8.4 \mathrm{~Hz})\end{array}$ & $6.37(\mathrm{~s})$ \\
\hline & $9 \mathbf{b}^{[9]} \mathrm{R}=\mathrm{CF}_{3}$ & $6.34(s)$ & $\begin{array}{c}7.26(\mathrm{~d} \\
J=6.9 \mathrm{~Hz})\end{array}$ & $\begin{array}{c}6.47(\mathrm{~d}, \\
J=8.4 \mathrm{~Hz})\end{array}$ & $6.37(\mathrm{~s})$ \\
\hline \multirow{2}{*}{ AcHN } & $10 \mathbf{a}^{[9]} \mathrm{R}=\mathrm{CH}_{3}$ & $6.26(\mathrm{~s})$ & $\begin{array}{c}7.62(\mathrm{~d}, \\
J=8.7 \mathrm{~Hz})\end{array}$ & $\begin{array}{c}7.30(\mathrm{~d}, \\
J=8.7 \mathrm{~Hz})\end{array}$ & 7.99 (s) \\
\hline & $\mathbf{1 0 b}^{[9]} \mathrm{R}=\mathrm{CF}_{3}$ & $6.79(\mathrm{~s})$ & $\begin{array}{c}7.62(\mathrm{~d}, \\
J=8.7 \mathrm{~Hz})\end{array}$ & $\begin{array}{c}7.35(\mathrm{~d} \\
J=9.0 \mathrm{~Hz})\end{array}$ & $7.98(\mathrm{~s})$ \\
\hline \multirow{2}{*}{$\mathrm{H}_{3} \mathrm{CO}$} & $11 a^{[8]} \mathrm{R}=\mathrm{CH}_{3}$ & $6.39(\mathrm{~s})$ & \multicolumn{2}{|c|}{-----7.13-7.18----- $(\mathrm{m})$} & $\begin{array}{c}7.26(\mathrm{dd}, J=1.8 \& \\
7.5 \mathrm{~Hz})\end{array}$ \\
\hline & $11 \mathbf{b}^{[9]} \mathrm{R}=\mathrm{CF}_{3}$ & $7.13(s)$ & \multicolumn{2}{|c|}{$-----7.48-7.82----(m)$} & $7.95(b r s)$ \\
\hline \multirow{2}{*}{ (N) } & $12 a^{[8]} \mathrm{R}=\mathrm{CH}_{3}$ & $6.36(\mathrm{~s})$ & \multicolumn{3}{|c|}{-----7.00-7.17-----(br s) } \\
\hline & $\mathbf{1 2}^{[9]} \mathrm{R}=\mathrm{CF}_{3}$ & 7.04 (s) & ----7.19 & -----(m) & $\begin{array}{c}7.95(\mathrm{~d} \\
J=6.6 \mathrm{~Hz})\end{array}$ \\
\hline \multirow{3}{*}{$\mathrm{AcO}_{\mathrm{R}}$} & $13 a^{[8]} \mathrm{R}=\mathrm{CH}_{3}$ & $6.46(\mathrm{~s})$ & -----7.29 & ---- (m) & $\begin{array}{c}7.47(\mathrm{dd}, J=1.5 \& \\
5.3 \mathrm{~Hz})\end{array}$ \\
\hline & $13 \mathbf{b}^{[9]} \mathrm{R}=\mathrm{CF}_{3}$ & $8.01(s)$ & $\begin{array}{c}7.98(\mathrm{~d} \\
J=2.1 \mathrm{~Hz})\end{array}$ & $\begin{array}{c}7.81(\mathrm{dd} \\
J=2.1 \& 9.0 \mathrm{~Hz})\end{array}$ & $\begin{array}{c}8.29(b r s, J=9.3 \\
H z)\end{array}$ \\
\hline & $14 \mathbf{a}^{[13]} \mathrm{R}=\mathrm{CH}_{3}$ & $6.17(\mathrm{~s})$ & $7.22(\mathrm{~s})$ & $7.53(d$ & $7.66(\mathrm{~d}$ \\
\hline
\end{tabular}




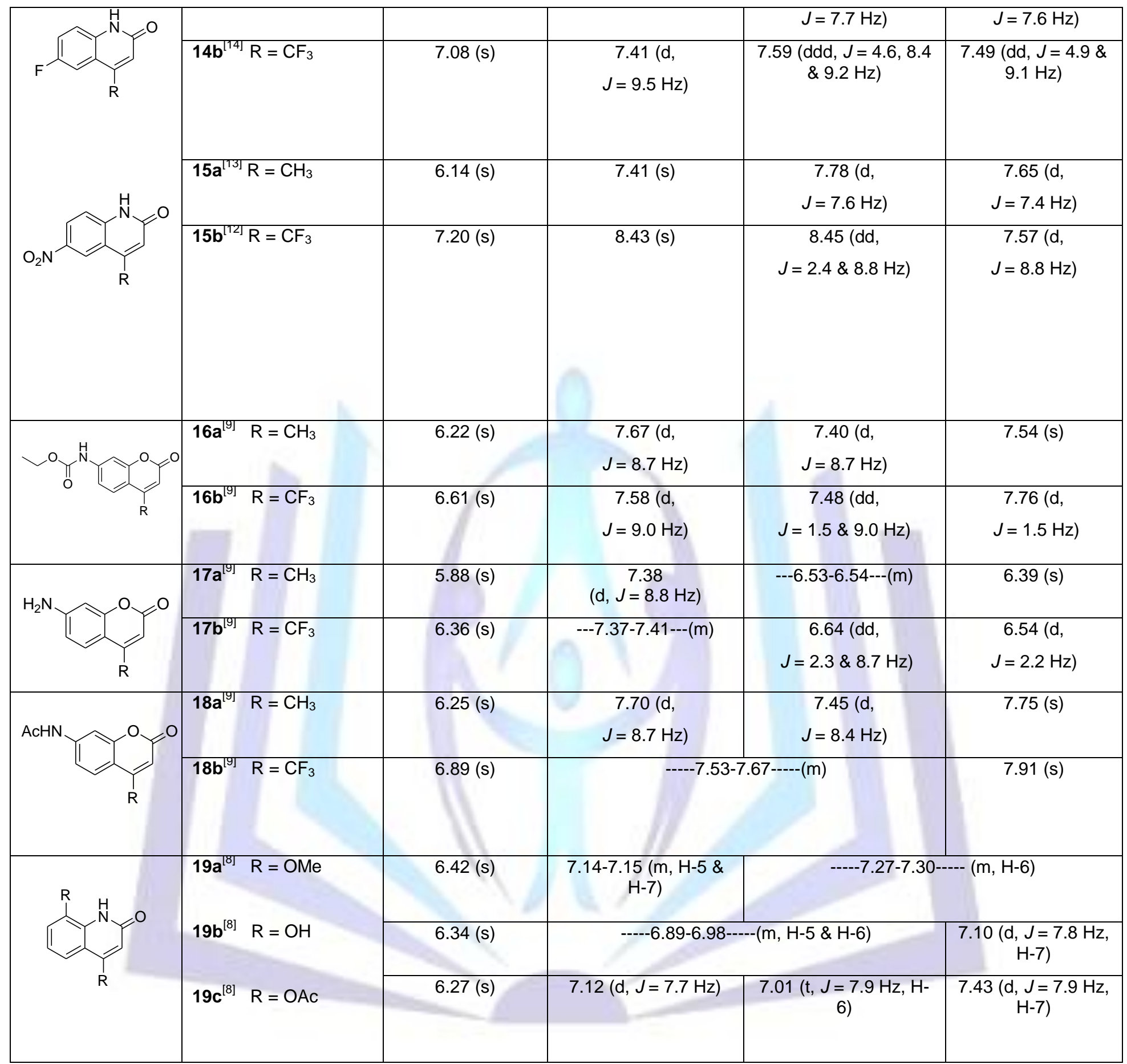

\section{EXPERIMENTAL}

In the case of compounds prepared in our laboratory, ${ }^{1} \mathrm{H}$ NMR was recorded on Bruker Avance-300 spectrometer in DMSO- $d_{6}$ as the solvent and TMS as internal reference. Solvent DMSO > 99.80\% pure, was from Aldrich.

Chemical shifts ( $\delta$ scale) are reported in parts per million $(\mathrm{ppm})$ relative to the central peak of the solvent, i.e. $2.5 \mathrm{ppm}$ for DMSO- $d_{6}$.

${ }^{1} \mathrm{H}$ NMR parameters are as follows: spectrometer frequency (SF), $300.13 \mathrm{MHz}$; acquisition time (AQ), $5.3084 \mathrm{~s}$; number of transients (NS), 16; receiver gain (RG), 287.4; temperature (TE), $300.0 \mathrm{~K}$; dwell time (DW), $81 \mu \mathrm{s}$; per scan delay (DE), $6.0 \mu \mathrm{s} ;$ dummy scans (DS), 2. 


\subsection{General procedure for the synthesis of methoxy-4-methylquinolin-2-ones}

Anisidine (20 g, $164 \mathrm{mmol})$ was added drop wise to trifluoromethyl/ethyl acetoacetate $(277 \mathrm{mmol})$ and the reaction mixture was refluxed for $20 \mathrm{~h}$. After the completion of reaction, the contents were cooled and then poured on sodium carbonate solution. The compound was extracted with chloroform and the solvent was evaporated in vacuo. $70 \%$ Sulphuric acid (40 $\mathrm{mL}$ ) was added and the reaction mixture was stirred at $95{ }^{\circ} \mathrm{C}$. The progress of reaction was monitored on TLC (5\% methanol-chloroform). On completion of the reaction, the solution was cooled and poured on crushed ice (500 g). The resulting precipitate was filtered and washed with water and petroleum ether. The crude product was recrystallized from ethanol to give methoxy quinolin-2-ones.

\subsubsection{7-Methoxy-4-methylquinolin-2(1H)-one (6a)}

It was obtained as white solid $(83 \%) ; \mathrm{mp}: 195{ }^{\circ} \mathrm{C}$, (Literature value $\left.=196{ }^{\circ} \mathrm{C}\right){ }^{15} ;{ }^{1} \mathrm{H}$ NMR $\left(\mathrm{DMSO}-d_{6}, 300 \mathrm{MHz}\right): \delta 2.45(\mathrm{~s}$ $\left.3 \mathrm{H}, \mathrm{C}-4 \mathrm{CH}_{3}\right), 3.88\left(\mathrm{~s}, 3 \mathrm{H}, \mathrm{OCH}_{3}\right), 6.30(\mathrm{~s}, 1 \mathrm{H}, \mathrm{H}-3), 6.84(\mathrm{~d}, 1 \mathrm{H}, J=8.7 \mathrm{~Hz}, \mathrm{H}-6), 6.94(\mathrm{~s}, 1 \mathrm{H}, \mathrm{H}-8), 7.66(\mathrm{~d}, 1 \mathrm{H}, J=8.9$ $\mathrm{Hz}, \mathrm{H}-5), 11.00$ (brs, $1 \mathrm{H}, \mathrm{NH}) ;{ }^{13} \mathrm{C}$ NMR (DMSO-d, $\left.75 \mathrm{MHz}\right): \delta 19.36\left(\mathrm{C}-4 \mathrm{CH}_{3}\right), 56.14\left(\mathrm{OCH}_{3}\right), 99.12,111.12(\mathrm{C}-6$ and C8), 114.68 (C-3), 118.56 (C-10), 127.02 (C-5), 141.26 (C-9), 148.85 (C-4), 161.80 (C-7), 162.89 (C-2); IR (KBr) V 2957.41, 1658.97, 1629.05, 1549.71, 1474.57, 1417.53, 1261.70, 1217.14, 1177.11, 1023.59, 856.63, 808.71, 710.74 cm ; $\mathrm{UV}$ (methanol) $\lambda_{\max }: 323$ and $337 \mathrm{~nm}$; HRMS: $\mathrm{C}_{11} \mathrm{H}_{11} \mathrm{O}_{2} \mathrm{~N}[\mathrm{M}]^{+}: 189.9665$.

\subsubsection{6-Methoxy-4-methylquinolin-2(1H)-one (11a)}

It was obtained as white solid $(75 \%) ; \mathrm{mp}: 260-262{ }^{\circ} \mathrm{C}$, (Literature value $\left.=260-262{ }^{\circ} \mathrm{C}\right){ }^{16} ;{ }^{1} \mathrm{H} \mathrm{NMR}\left(\mathrm{DMSO}-d_{6}, 300 \mathrm{MHz}\right): \delta$ $2.42\left(\mathrm{~s}, 3 \mathrm{H}, \mathrm{C}-4 \mathrm{CH}_{3}\right), 3.82\left(\mathrm{~s}, 3 \mathrm{H}, \mathrm{OCH}_{3}\right), 6.39(\mathrm{~s}, 1 \mathrm{H}, \mathrm{H}-3), 7.13-7.18(\mathrm{~m}, 2 \mathrm{H}, \mathrm{H}-5$ and H-7), $7.26(\mathrm{dd}, 1 \mathrm{H}, J=1.8 \& 5.3$ $\mathrm{Hz}, \mathrm{H}-8), 11.46$ (brs, $1 \mathrm{H}, \mathrm{NH}) ;{ }^{13} \mathrm{C}$ NMR (DMSO-d, $\left.75 \mathrm{MHz}\right): \delta 19.45\left(\mathrm{C}-4 \mathrm{CH}_{3}\right), 56.37\left(\mathrm{OCH}_{3}\right), 107.69,117.52(\mathrm{C}-5 \mathrm{and}$ C-7), 119.87 (C-10), 121.06 (C-8), 122.11 (C-9), 131.38 (C-3), 148.27 (C-4), 154.96 (C-6), 162.03 (C-2); IR (KBr) V $\operatorname{max:~}$ 3433.06, 2821.76, 1653.70, 1621.98, 1503.71, 1421.31, 1275.63, 1240.22, 1202.18, 1179.30, 1044.05, 835.98, 628.40 $\mathrm{cm}^{-1}$; UV (methanol) $\lambda_{\max }: 269$ and $350 \mathrm{~nm}$; HRMS: $\mathrm{C}_{11} \mathrm{H}_{11} \mathrm{O}_{2} \mathrm{~N}[\mathrm{M}]^{+}: 189.8188$.

\subsubsection{8-Methoxy-4-methylquinolin-2(1H)-one (19a)}

It was obtained as white solid (30\%); mp: 192-194 ${ }^{\circ} \mathrm{C}$, (Literature value $\left.=188-190{ }^{\circ} \mathrm{C}\right){ }^{17} ;{ }^{1} \mathrm{H} \mathrm{NMR}\left(\mathrm{DMSO}-d_{6}, 300 \mathrm{MHz}\right): \delta$ $2.41\left(\mathrm{~s}, 3 \mathrm{H}, \mathrm{C}-4 \mathrm{CH}_{3}\right), 3.89\left(\mathrm{~s}, 3 \mathrm{H}, \mathrm{OCH}_{3}\right), 6.42(\mathrm{~s}, 1 \mathrm{H}, \mathrm{H}-3), 7.14-7.15(\mathrm{~m}, 2 \mathrm{H}, \mathrm{H}-5$ and H-7), 7.27-7.30 (m, 1H, H-6), 10.58 (brs, $1 \mathrm{H}, \mathrm{NH}) ;{ }^{13} \mathrm{C}$ NMR (DMSO- $\left.d_{6}, 75 \mathrm{MHz}\right): \delta 18.74\left(\mathrm{C}-4 \mathrm{CH}_{3}\right), 56.07\left(\mathrm{OCH}_{3}\right), 110.89,116.36(\mathrm{C}-5$ and $\mathrm{C}-7)$, 120.08 (C-9), 121.36, 121.57 (C-3 and C-6), 128.57 (C-10), 145.80 (C-4), 148.15 (C-8), 161.14 (C-2); IR (KBr) Vmax: 3163.95, 2933.15, 1648.26, 1605.82, 1462.84, 1389.90, 1265.97, 1154.88, 1049.33, 860.83, 791.04, 739.93, 726.00, $636.77 \mathrm{~cm}^{-1}$; UV (methanol) $\lambda_{\max }: 278$ and $335 \mathrm{~nm}$; HRMS: $\mathrm{C}_{11} \mathrm{H}_{11} \mathrm{O}_{2} \mathrm{~N}[\mathrm{M}]^{+}: 189.7739$.

\subsubsection{6-Methoxy-4-(trifluoromethyl)quinolin-2(1H)-one (11b)}

The product was obtained through column chromatography in ethyl acetate/petroleum ether (1:49). The title compound (11b) was obtained as yellow solid in $70 \%$ yield by following the general procedure. Melting point: $270^{\circ} \mathrm{C} ;{ }^{1} \mathrm{H}$ NMR (DMSO- $\left.\mathrm{d}_{6}, 300 \mathrm{MHz}\right): \delta 3.72(\mathrm{~s}, 3 \mathrm{H},-\mathrm{OCH} 3), 7.13(\mathrm{~s}, 1 \mathrm{H}, \mathrm{H}-3), 7.48-7.82(\mathrm{~m}, 2 \mathrm{H}, \mathrm{H}-5$ and $\mathrm{H}-7), 7.95(\mathrm{brs}, 1 \mathrm{H}, \mathrm{H}-8)$ and 12.14 (brs, 1H, NH); 13C NMR (DMSO-d, $75.5 \mathrm{MHz})$ : $\delta 55.53$ (-OCH3), 99.12, 100.01 (C-7 and C-5), 114.68 (C-3), 122.18 and 123.69 (C-8 and C-9), 130.67 (C-10), 143.96 (C-4), 144.06-145.09 (-CF $), 157.89$ (C-6) and 161.55 (C-2); HRMS: Calculated for $\mathrm{C}_{11} \mathrm{H}_{8} \mathrm{~F}_{3} \mathrm{NO}_{2}[\mathrm{M}]^{+}$243.1819, found 243.5345 .

\section{7-Methoxy-4-(trifluoromethyl)quinolin-2(1H)-one (6b)}

The title compound $(\mathbf{6 b})$ was obtained through column chromatography in ethyl acetate/petroleum ether (1:49). It was obtained by as yellow solid in $80 \%$ yield following the general procedure. Melting point: $250-252^{\circ} \mathrm{C},{ }^{1} \mathrm{H}$ NMR (DMSO-d 6 , $300 \mathrm{MHz}): \delta 3.84(\mathrm{~s}, 3 \mathrm{H},-\mathrm{OCH}), 6.77(\mathrm{~s}, 1 \mathrm{H}, \mathrm{H}-3), 6.92-6.96(\mathrm{~m}, 2 \mathrm{H}, \mathrm{H}-6$ and $\mathrm{H}-8), 7.61(\mathrm{~d}, 1 \mathrm{H}, \mathrm{J}=8.4 \mathrm{~Hz}, \mathrm{H}-5)$ and 12.20 (brs, 1H, NH); 13C NMR (DMSO-d, $75.5 \mathrm{MHz}$ ): $\delta 55.54$ (-OCH3), 99.79, 107.01 (C-6 and C-8), 112.00 (C-3), 118.13 (C-10), 124.37 (C-5), 125.64 (C-9), 136.23-136.64 (-CF3), 141.75 (C-4), 160.38 (C-7) and 161.63 (C-2); HRMS Calculated for $\mathrm{C}_{11} \mathrm{H}_{8} \mathrm{~F}_{3} \mathrm{NO}_{2}[\mathrm{M}+\mathrm{H}]^{+} 244.1819$, found 244.3801 .

\subsection{General procedure for the synthesis of hydroxy-4-methylquinolin-2-ones}

A mixture of hydrobromic acid and acetic acid (7:3) was added to methoxy-4-methyl-1H-quinolin-2-ones. The reaction mixture was refluxed for $75 \mathrm{~h}$ and then poured on crushed ice. The resulting precipitate was filtered and washed with water to yield 3-alkyl-7-hydroxyquinolin-2-ones. ${ }^{18}$

\subsubsection{7-Hydroxy-4-methylquinolin-2(1H)-one (7a)}

It was obtained as white solid (85\%); mp: $>300{ }^{\circ} \mathrm{C}$, (Literature value $\left.=306-307{ }^{\circ} \mathrm{C}\right){ }^{19} ;{ }^{1} \mathrm{H} \mathrm{NMR}\left(\mathrm{DMSO}-d_{6}, 500 \mathrm{MHz}\right): \delta$ $2.28\left(\mathrm{~s}, 3 \mathrm{H}, \mathrm{C}-4 \mathrm{CH}_{3}\right), 6.08(\mathrm{~s}, 1 \mathrm{H}, \mathrm{H}-3), 6.59(\mathrm{~d}, 1 \mathrm{H}, \mathrm{J}=8.8 \mathrm{~Hz}, \mathrm{H}-6), 6.65(\mathrm{~d}, 1 \mathrm{H}, \mathrm{J}=2.4 \mathrm{~Hz}, \mathrm{H}-8), 7.45(\mathrm{~d}, 1 \mathrm{H}, J=8.8$ $\mathrm{Hz}, \mathrm{H}-5), 10.02$ (brs, $1 \mathrm{H}, \mathrm{OH}), 11.33$ (brs, $1 \mathrm{H}, \mathrm{NH}) ;{ }^{13} \mathrm{C}$ NMR (DMSO-d $\left.6,125 \mathrm{MHz}\right): \delta 19.03\left(\mathrm{C}-4 \mathrm{CH}_{3}\right), 100.61,111.80(\mathrm{C}-$ 6 and C-8), 113.32 (C-3), 117.50 (C-10), 126.68 (C-5), 141.04 (C-9), 148.44 (C-4), 159.95 (C-7), 162.69 (C-2); IR (KBr) $V_{\text {max }}$ : 3425.24, 2927.52, 2364.39, 1652.89, 1541.56 (amide-II), 1474.84, 1406.35, 1255.85, 1220.96, 1074.07, 905.44, 817.60, $689.94 \mathrm{~cm}^{-1}$; UV (methanol) $\lambda_{\max }: 324$ and $337 \mathrm{~nm}$; HRMS: $\mathrm{C}_{10} \mathrm{H}_{9} \mathrm{O}_{2} \mathrm{~N}[\mathrm{M}+\mathrm{H}]^{+}: 176.2008$. 


\subsubsection{6-Hydroxy-4-methylquinolin-2(1H)-one (12a)}

It was obtained as white solid (80\%); mp: $324-326{ }^{\circ} \mathrm{C}$, (Literature value $\left.=326-330{ }^{\circ} \mathrm{C}\right)^{20} ;{ }^{1} \mathrm{H} \mathrm{NMR}\left(\mathrm{DMSO}-d_{6}, 300 \mathrm{MHz}\right): \delta$ 2.34 (s, 3H, C-4 CH$~_{3}$ ), 6.36 (s, $\left.1 \mathrm{H}, \mathrm{H}-3\right), 7.00$ (brs, $2 \mathrm{H}, \mathrm{H}-5$ and $\left.\mathrm{H}-7\right), 7.17$ (brs, $\left.1 \mathrm{H}, \mathrm{H}-8\right), 9.40$ (brs, $\left.1 \mathrm{H}, \mathrm{OH}\right), 11.42$ (brs, $1 \mathrm{H}, \mathrm{NH}) ;{ }^{13} \mathrm{C}$ NMR (DMSO- $\left.d_{6}, 75 \mathrm{MHz}\right): \delta 19.36\left(\mathrm{C}-4 \mathrm{CH}_{3}\right), 109.41,117.43(\mathrm{C}-5$ and $\mathrm{C}-7), 120.39(\mathrm{C}-10), 121.09(\mathrm{C}-8)$, 121.80 (C-9), 132.74 (C-3), 148.01 (C-4), 152.93 (C-6), 161.99 (C-2); IR (KBr) $v_{\text {max }}: 3489.59,3187.46,1661.73,1634.87$, 1608.22, 1513.42, 1433.01, 1294.34, 1191.88, 866.12, 853.10, 810.02, $642.28 \mathrm{~cm}^{-1}$; UV (methanol) $\lambda_{\max }: 269$ and $352 \mathrm{~nm}$; HR MS: $\mathrm{C}_{10} \mathrm{H}_{9} \mathrm{O}_{2} \mathrm{~N}[\mathrm{M}]^{+}: 175.7805$.

\subsubsection{8-Hydroxy-4-methylquinolin-2(1H)-one (19b)}

It was obtained as white solid (70\%); mp: $250-254{ }^{\circ} \mathrm{C}$, (Literature value $\left.=248{ }^{\circ} \mathrm{C}\right)^{21} ;{ }^{1} \mathrm{H}$ NMR $\left(\mathrm{DMSO}-d_{6}, 300 \mathrm{MHz}\right): \delta 2.37$ (s, 3H, C-4 $\left.\mathrm{CH}_{3}\right), 6.34(\mathrm{~s}, 1 \mathrm{H}, \mathrm{H}-3), 6.89-6.98(\mathrm{~m}, 2 \mathrm{H}, \mathrm{H}-5$ and $\mathrm{H}-6), 7.10(\mathrm{~d}, 1 \mathrm{H}, \mathrm{J}=7.8 \mathrm{~Hz}, \mathrm{H}-7), 10.25(b r s, 2 \mathrm{H}, \mathrm{NH}$ and $\mathrm{OH}) ;{ }^{13} \mathrm{C}$ NMR (DMSO- $\left.d_{6}, 75 \mathrm{MHz}\right): \delta 18.70\left(\mathrm{C}-4 \mathrm{CH}_{3}\right), 114.36,114.96$ (C-5 and C-7), $120.48(\mathrm{C}-9), 121.04,121.60(\mathrm{C}-3$ and C-6), 127.84 (C-10), 143.67 (C-4), 148.19 (C-8), 160.91 (C-2); IR (KBr) $V_{\text {max }}$ : 3401.25, 1639.37, 1601.19, 1553.68, 1478.15, 1398.60, 1288.58, 1208.23, 1145.95, 924.47, 861.73, 792.88, 735.68, 600.31 $\mathrm{cm}^{-1}$; UV (methanol) $\lambda_{\max }: 254,279$ and $336 \mathrm{~nm}$; HRMS: $\mathrm{C}_{10} \mathrm{H}_{9} \mathrm{O}_{2} \mathrm{~N}[\mathrm{M}]^{+}: 175.9968$.

\subsubsection{6-Hydroxy-4-(trifluoromethyl)quinolin-2(1H)-one (12b)}

The title compound (12b) was obtained as yellow solid in $70 \%$ yield by following the general procedure. Melting point: 268 ${ }^{\circ} \mathrm{C} ;{ }^{1} \mathrm{H}$ NMR (DMSO-d, $\left.300 \mathrm{MHz}\right): \delta 7.04(\mathrm{~s}, 1 \mathrm{H}, \mathrm{H}-3), 7.19-7.34(\mathrm{~m}, 2 \mathrm{H}, \mathrm{H}-5$ and H-7), $7.95(\mathrm{~d}, 1 \mathrm{H}, \mathrm{J}=6.6 \mathrm{~Hz}, \mathrm{H}-8)$, 10.29 (brs, $1 \mathrm{H}, \mathrm{OH}$ ), 11.96 (brs, $1 \mathrm{H}, \mathrm{NH}) ; 13 \mathrm{C}$ NMR (DMSO-d, $75.5 \mathrm{MHz})$ : $\delta 99.54$ and 102.94 (C-5 and C-7), 119.95 (C3), 122.69 and 123.45 (C-8 and C-9), 130.70 (C-10), 143.04 (C-4), 144.02-144.48 (-CF 3 ), 156.27 (C-6), 161.11 (C-2); HRMS: Calculated for $\mathrm{C}_{10} \mathrm{H}_{6} \mathrm{~F}_{3} \mathrm{NO}_{2}[\mathrm{M}+\mathrm{H}]^{+} 230.0423$, found 230.0424 .

\subsubsection{7-Hydroxy-4-(trifluoromethyl)quinolin-2(1H)-one (7b)}

The title compound (7b) was obtained as yellow solid in $70 \%$ yield by following the general procedure. Melting point: $>300$ ${ }^{\circ} \mathrm{C} ;{ }^{1} \mathrm{H}$ NMR (DMSO-d, $\left.300 \mathrm{MHz}\right): \delta 6.68(\mathrm{~s}, 1 \mathrm{H}, \mathrm{H}-3), 6.76-6.82(\mathrm{~m}, 2 \mathrm{H}, \mathrm{H}-5$ and $\mathrm{H}-8), 7.53(\mathrm{dd}, 1 \mathrm{H}, \mathrm{J}=2.1 \mathrm{land} 8.7 \mathrm{~Hz}$, $\mathrm{H}-6), 10.57$ (brs, $1 \mathrm{H}, \mathrm{OH}$ ) and 12.08 (brs, $1 \mathrm{H}, \mathrm{NH}$ ); 13C NMR (DMSO-d, $75.5 \mathrm{MHz}$ ): $\delta 100.70$ and 106.05 (C-6 and C-8), 112.89 (C-3), 117.04 (C-10), 120.80 (C-5), 124.44 (C-9), 125.75 (C-4), 136.34-136.75 (-CF $), 141.86$ (C-7) and 160.45 (C2); HRMS: Calculated for $\mathrm{C}_{10} \mathrm{H}_{6} \mathrm{~F}_{3} \mathrm{NO}_{2}[\mathrm{M}+\mathrm{H}]^{+} 230.0423$, found 230.0428 .

\subsection{General procedure for the synthesis of 4-methyl-2-oxo-1,2-dihydroquinolin-yl acetate}

A solution of acetic anhydride and acetic acid (1:4) was added to 3-alkyl-hydroxy-4-methylquinolin-2(1H)-ones 10-18. The reaction mixture was refluxed for $6 \mathrm{hr}$ and then poured on crushed ice. The resulting precipitate was filtered and washed with water to yield acetoxy-3-alkylquinolin-2-ones.

\subsubsection{4-Methyl-2-oxo-1,2-dihydroquinolin-7-yl acetate (8a)}

It was obtained as white solid (90\%); mp: $258{ }^{\circ} \mathrm{C}$, (Literature value $\left.=257-258{ }^{\circ} \mathrm{C}\right) ;{ }^{1} \mathrm{H}$ NMR $\left(\mathrm{DMSO}-d_{6}, 500 \mathrm{MHz}\right): \delta 2.30$

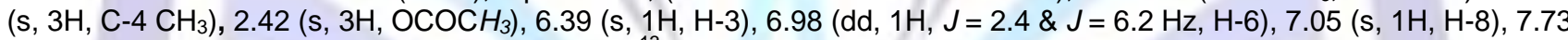
$(\mathrm{d}, 1 \mathrm{H}, J=6.1 \mathrm{~Hz}, \mathrm{H}-5), 11.67$ (brs, $1 \mathrm{H}, \mathrm{NH}) ;{ }^{13} \mathrm{C}$ NMR (DMSO-d, $\left.125 \mathrm{MHz}\right): \delta 19.38\left(\mathrm{C}_{-4} \mathrm{CH}_{3}\right), 21.76\left(\mathrm{OCOCH}_{3}\right)$, 108.77, 116.71 (C-6 and C-8), 118.36 (C-3), 121.21 (C-10), 126.98 (C-5), 140.43 (C-9), 148.52 (C-4), 152.59 (C-7), $162.63(\mathrm{C}-2), 169.82\left(-\mathrm{OCOCH}_{3}\right)$; IR (KBr) $\mathrm{V}_{\max }: 2927.12,2855.27,1751.47,1678.55,1561.24,1510.88,1458.53$, 1362.63, 1232.32, $1166.29,1025.06,906.26,856.71,645.56 \mathrm{~cm}^{-1}$; UV (methanol) $\lambda_{\max }: 323$ and $335 \mathrm{~nm}$; HRMS: $\mathrm{C}_{12} \mathrm{H}_{11} \mathrm{O}_{3} \mathrm{~N}[\mathrm{M}]^{+}: 217.2915$

\subsubsection{4-Methyl-2-oxo-1,2-dihydroquinolin-6-yl acetate (13a)}

It was obtained as white solid (85\%); mp: $270{ }^{\circ} \mathrm{C} ;{ }^{1} \mathrm{H}$ NMR (DMSO-d, $300 \mathrm{MHz}$ ): $\delta 2.30$ (s, $\left.3 \mathrm{H}, \mathrm{C}-4 \mathrm{CH}_{3}\right), 2.40$ (s, $3 \mathrm{H}$, $\left.\mathrm{OCOCH}_{3}\right), 6.46(\mathrm{~s}, 1 \mathrm{H}, \mathrm{H}-3), 7.29-7.35(\mathrm{~m}, 2 \mathrm{H}, \mathrm{H}-5$ and H-7), $7.17(\mathrm{dd}, 1 \mathrm{H}, J=1.5 \& 7.5 \mathrm{~Hz}, \mathrm{H}-8), 11.71($ brs, $1 \mathrm{H}, \mathrm{NH})$ ${ }^{13} \mathrm{C}$ NMR (DMSO-d $\left.d_{6}, 75 \mathrm{MHz}\right): \delta 18.39\left(\mathrm{C}-4 \mathrm{CH}_{3}\right), 20.76\left(\mathrm{OCOCH}_{3}\right), 116.27,117.06(\mathrm{C}-5$ and C-7), $120.01(\mathrm{C}-10), 121.47$ (C-8), 124.63 (C-3), 136.38 (C-9), 144.72 (C-4), 147.43 (C-6), 161.45 (C-2), $169.53\left(\mathrm{OCOCH}_{3}\right)$; IR (KBr) $\mathrm{V}_{\max }: 3433.37$, 2835.40, 1751.37, 1656.34, 1559.57, 1502.72, 1425.27, 1373.09, 1212.40, 1166.23, 1135.37, 1012.90, 905.94, 871.27, 842.91, $683.29 \mathrm{~cm}^{-1}$; UV (methanol) $\lambda_{\max }: 264$ and $334 \mathrm{~nm}$; HRMS: $\mathrm{C}_{12} \mathrm{H}_{11} \mathrm{O}_{3} \mathrm{~N}[\mathrm{M}+\mathrm{H}]^{+}: 218.4582$.

\subsubsection{4-Methyl-2-oxo-1,2-dihydroquinolin-8-yl acetate (19c)}

It was obtained as white solid (85\%); mp: $242-244{ }^{\circ} \mathrm{C} ;{ }^{1} \mathrm{H}$ NMR (DMSO-d, $300 \mathrm{MHz}$ ): $\delta 2.19$ (s, $3 \mathrm{H}, \mathrm{C}-4 \mathrm{CH}$ ), 2.25 (s, $\left.3 \mathrm{H}, \mathrm{OCOCH}_{3}\right), 6.27(\mathrm{~s}, 1 \mathrm{H}, \mathrm{H}-3), 7.01(\mathrm{t}, 1 \mathrm{H}, J=7.9 \mathrm{~Hz}, \mathrm{H}-6), 7.12(\mathrm{~d}, 1 \mathrm{H}, J=7.7 \mathrm{~Hz}, \mathrm{H}-5), 7.43(\mathrm{~d}, 1 \mathrm{H}, J=7.9 \mathrm{~Hz}, \mathrm{H}-7)$, 11.27 (brs, $1 \mathrm{H}, \mathrm{NH}) ;{ }^{13} \mathrm{C}$ NMR (DMSO-d, $\left.75 \mathrm{MHz}\right): \delta 18.68\left(\mathrm{C}-4 \mathrm{CH}_{3}\right), 21.35\left(\mathrm{OCOCH}_{3}\right), 121.19(\mathrm{C}-9), 121.23,121.48$ (C-7 and C-3), 122.36, 123.84 (C-5 and C-6), 131.54 (C-10), 136.74 (C-4), 148.00 (C-8), $161.63(\mathrm{C}-2), 169.64\left(\mathrm{OCOCH}_{3}\right)$ IR $(\mathrm{KBr}) \mathrm{V}_{\max }: 3431.86,2996.30,1763.12,1671.14,1647.83,1605.69,1475.41,1422.01,1366.81,1195.13,1168.09$, 1138.04, 1016.39, 932.32, 869.41, 744.18, $689.19 \mathrm{~cm}^{-1}$; UV (methanol) $\lambda_{\max }: 268$ and $328 \mathrm{~nm} ; \mathrm{HRMS} \mathrm{C}_{12} \mathrm{H}_{11} \mathrm{O}_{3} \mathrm{~N}[\mathrm{M}+\mathrm{H}]^{+}$ 218.1072 


\subsubsection{2-0xo-4-(trifluoromethyl)-1,2-dihydro-6-yl acetate (13b)}

The title compound (13b) was obtained as white crystals in $90 \%$ yield by following the general procedure. Melting point: $296{ }^{\circ} \mathrm{C} ; 1 \mathrm{H}$ NMR (DMSO-d $\left.6,300 \mathrm{MHz}\right): \delta 2.37\left(\mathrm{~s}, 3 \mathrm{H},-\mathrm{OCOCH}_{3}\right), 7.81(\mathrm{dd}, 1 \mathrm{H}, \mathrm{J}=2.1$ and $9.0 \mathrm{~Hz}, \mathrm{H}-7), 7.98(\mathrm{~d}, 1 \mathrm{H}, \mathrm{J}=$ $2.1 \mathrm{~Hz}, \mathrm{H}-5), 8.01$ (s, $1 \mathrm{H}, \mathrm{H}-3), 8.29$ (brs, $1 \mathrm{H}, \mathrm{J}=9.3 \mathrm{~Hz}, \mathrm{H}-8) ; 13 \mathrm{C}$ NMR (DMSO-d, $75.5 \mathrm{MHz}): \delta 20.88\left(-\mathrm{OCOCH}_{3}\right)$ $110.36,113.19$ (C-7 and C-5), 119.30 (C-3), 123.53 (C-8), 128.06 (C-9), 131.20 (C-10), 146.23-147.18 (-CF $), 150.65(\mathrm{C}-$ 4), $155.69(\mathrm{C}-6), 168.24(\mathrm{C}-2)$ and $169.05\left(-\mathrm{OCOCH}_{3}\right)$; HRMS: Calculated for $\mathrm{C}_{12} \mathrm{H}_{8} \mathrm{~F}_{3} \mathrm{NO}_{3}[\mathrm{M}+\mathrm{H}]^{+}$272.0456, found 271.9899

\subsubsection{2-0xo-4-(trifluoromethyl)-1,2-dihydro-7-yl acetate (13b)}

The title compound (13b) was obtained as white crystals in $90 \%$ yield by following the general procedure. Melting point: $190 \circ \mathrm{C} ;{ }^{1} \mathrm{H}$ NMR (DMSO-d $\left.6,300 \mathrm{MHz}\right): \delta 2.32\left(\mathrm{~s}, 3 \mathrm{H},-\mathrm{OCOCH}_{3}\right), 6.97(\mathrm{~s}, 1 \mathrm{H}, \mathrm{H}-3), 7.12(\mathrm{dd}, 1 \mathrm{H}, \mathrm{J}=1.8 \mathrm{and} 8.7 \mathrm{~Hz}, \mathrm{H}-6)$, $7.19(\mathrm{~d}, 1 \mathrm{H}, \mathrm{J}=1.8 \mathrm{~Hz}, \mathrm{H}-8)$ and $7.73(\mathrm{~d}, 1 \mathrm{H}, \mathrm{J}=8.7 \mathrm{~Hz}, \mathrm{H}-5) ;{ }^{13} \mathrm{C}$ NMR (DMSO-d, $\left.75.5 \mathrm{MHz}\right): \delta 20.98\left(-\mathrm{OCOCH}_{3}\right)$, 108.94, 110.91 (C- 6 and C-8), 117.40 (C-3), 121.45 (C-10), 124.27 (C-5), 125.72 (C-9), 136.00-136.42 (CF $), 140.84$ (C4), $152.56(\mathrm{C}-7), 160.15(\mathrm{C}-2)$ and $168.84\left(-\mathrm{OCOCH}_{3}\right)$; HRMS: Calculated for $\mathrm{C}_{12} \mathrm{H}_{8} \mathrm{~F}_{3} \mathrm{NO}_{3}$ [M]+. 271.1920, found 271.3652

\subsubsection{4-Hydroxyquinolin-2(1H)-one (3)}

It was obtained as yellow solid (70\%); mp: $320{ }^{\circ} \mathrm{C}$, (Literature value $\left.=318-320{ }^{\circ} \mathrm{C}\right) ;{ }^{1} \mathrm{H}$ NMR $\left(\mathrm{DMSO}-d_{6}, 300 \mathrm{MHz}\right): \delta 5.76$ $(s, 1 \mathrm{H}, \mathrm{H}-3), 7.11-7.78(\mathrm{~m}, 4 \mathrm{H}, \mathrm{H}-5, \mathrm{H}-6, \mathrm{H}-7$ and $\mathrm{H}-8), 11.18(\mathrm{brs}, 1 \mathrm{H}, \mathrm{OH}) ;{ }^{13} \mathrm{C}$ NMR (DMSO-d, $\left.75 \mathrm{MHz}\right): \delta 98.18(\mathrm{C}-3)$, 114.95, 115.10 (C-6 and C-8), 121.04 (C-5), 122.62 (C-10), 130.82 (C-7), 139.13 (C-9), 162.43 (C-4), 163.57 (C-2); IR $(\mathrm{KBr}) \mathrm{v}_{\max }: 3430.12,3094.44,2953.09,2861.12,2639.30,2364.36,1669.52,1633.94,1594.78,1560.44,1506.18$, $1471.81,1420.44,1378.93,1332.01,1259.95,1235.09,1160.89,1145.18,1102.39,1035.00,909.53,867.70,773.96$, 762.96, 755.55, 671.97, 630.01 $\mathrm{cm}^{-1}$; UV (methanol) $\lambda_{\max }: 269$ and $314 \mathrm{~nm}$; HRMS: $\mathrm{C}_{9} \mathrm{H}_{7} \mathrm{O}_{2} \mathrm{~N}[\mathrm{M}]^{+}: 161.7715$.

\subsubsection{2-Oxo-1,2-dihydroquinolin-4-yl acetate (4)}

It was obtained as yellow solid (85\%); mp: $220{ }^{\circ} \mathrm{C}$, (Literature value $=217-219{ }^{\circ} \mathrm{C}$ ); ${ }^{1} \mathrm{H}$ NMR (DMSO- $d_{6}, 300 \mathrm{MHz}$ ): $\delta \square 2.45$ $\left(\mathrm{s}, 3 \mathrm{H},-\mathrm{OCOCH}_{3}\right), 6.65(\mathrm{~s}, 1 \mathrm{H}, \mathrm{H}-3), 7.22-7.66(\mathrm{~m}, 4 \mathrm{H}, \mathrm{H}-5, \mathrm{H}-6, \mathrm{H}-7$ and $\mathrm{H}-8), 11.89(\mathrm{brs}, 1 \mathrm{H}, \mathrm{NH}) ;{ }^{13} \mathrm{C} \mathrm{NMR}\left(\mathrm{DMSO}-d_{6}\right.$, $75 \mathrm{MHz}): \delta 20.75\left(-\mathrm{OCOCH}_{3}\right), 98.19$ (C-3), 112.28, 115.48 (C-6 and C-8), 122.03 (C-5), 122.44 (C-10), 131.47 (C-7), 138.87 (C-9), 156.04 (C-4), 162.39 (C-2), $172.04\left(-\mathrm{OCOCH}_{3}\right)$; IR (KBr) $\mathrm{v}_{\max }: 3396.31,3009.90,2965.78,2858.41$, 1768.58, 1668.77, 1615.91, 1561.20, 1506.34, 1475.03, 1434.55, 1398.53, 1370.26, 1268.81, 1189.23, 1160.47, 1143.99, 1078.60, 984.19, 953.88, 887.47, 770.84, 758.32, 660.04 cm $\mathrm{cm}^{-1}$; UV (methanol) $\lambda_{\max }: 264$ and $326 \mathrm{~nm} ; \mathrm{HRMS}: \mathrm{C}_{11} \mathrm{H}_{9} \mathrm{O}_{3} \mathrm{~N}$ $[\mathrm{M}+\mathrm{H}]^{+}: 204.1366$.

\subsection{General procedure for the synthesis of 7-aminoquinolin-2(1H)-one}

1,3-Diaminobenzene $(1.0 \mathrm{~g}, 9.3 \mathrm{mmol})$, was added to substituted ethyl acetoacetates $(1.2$ equivalent, 2-3, 6) and the mixture was refluxed for $20 \mathrm{~h}$. It was then poured on ice $(100 \mathrm{~g})$ and the precipitate was filtered. The product was obtained through column chromatography using silica gel (100-200 mesh) in methanol/chloroform (1:99).

\subsubsection{7-Amino-4-methylquinolin-2(1H)-one (9a)}

The title compound (9a) was obtained as yellow solid in $65 \%$ yield by following the above general procedure. Melting point: $271-272{ }^{\circ} \mathrm{C}$ (Literature value $\left.=271{ }^{\circ} \mathrm{C}\right) ;{ }^{1} \mathrm{H}$ NMR (DMSO-d $\left.6,300 \mathrm{MHz}\right)$ : $\delta 2.28(\mathrm{~s}, 3 \mathrm{H}, \mathrm{C}-4 \mathrm{CH} 3), 5.74(\mathrm{brs}, 2 \mathrm{H}$, $\mathrm{NH} 2), 5.96(\mathrm{~s}, 1 \mathrm{H}, \mathrm{H}-3), 6.37(\mathrm{~s}, 1 \mathrm{H}, \mathrm{H}-8), 6.46(\mathrm{~d}, 1 \mathrm{H}, \mathrm{J}=8.4 \mathrm{~Hz}, \mathrm{H}-6), 7.34(\mathrm{~d}, 1 \mathrm{H}, \mathrm{J}=8.7 \mathrm{~Hz}, \mathrm{H}-5), 11.18(\mathrm{brs}, 1 \mathrm{H}, \mathrm{NH})$; 13C NMR (DMSO-d 6 , 75.5 MHz): $\delta 18.55$ (C-4 CH3), 96.85, 110.48, 110.55 and 114.67 (C-3, C-6, C-8 and C-10), 125.67 (C-5), 140.81 (C-9), 148.13 (C-4), 151.15 (C-7), 162.55 (C-2); ESI MS: Calculated for $\mathrm{C}_{10} \mathrm{H}_{10} \mathrm{~N}_{20}$ [M] $]^{+} 174$, found 174.

\subsection{2. $\quad \mathrm{N}$-(4-Methyl-2-oxo-1,2-dihydroquinolin-7-yl) acetamide (10a)}

The title compound (10a) was obtained as brown crystals in $60 \%$ yield by following the general procedure. Melting point: $300^{\circ} \mathrm{C}$ (Literature value $\left.=300{ }^{\circ} \mathrm{C}\right) ;{ }^{1} \mathrm{H}$ NMR $($ DMSO-d, $300 \mathrm{MHz}): \delta 2.09\left(\mathrm{~s}, 3 \mathrm{H}, \mathrm{H}-2{ }^{2}\right), 2.38(\mathrm{~s}, 3 \mathrm{H}, \mathrm{C}-4 \mathrm{CH} 3), 6.26(\mathrm{~s}$, $1 \mathrm{H}, \mathrm{H}-3), 7.30(\mathrm{~d}, 1 \mathrm{H}, \mathrm{J}=8.7 \mathrm{~Hz}, \mathrm{H}-6), 7.62(\mathrm{~d}, 1 \mathrm{H}, \mathrm{J}=8.7 \mathrm{~Hz}, \mathrm{H}-5), 7.99(\mathrm{~s}, 1 \mathrm{H}, \mathrm{H}-8), 10.197,11.53(2 \times \mathrm{brs}, 2 \mathrm{H}, 2 \times$ NHCO); 13C NMR (DMSO-d, $75.5 \mathrm{MHz}): \delta 18.44(\mathrm{C}-4 \mathrm{CH} 3), 24.18$ (C-2'), 104.37 and 113.48 (C-6 and C-8), 115.46 and 118.89 (C-3 and C-10), 125.31 (C-5), 139.51 (C-9), 141.05 (C-4), 147.74 (C-7), 162.14 (C-2), 168.85 (C-1'); ESI MS: Calculated for $\mathrm{C}_{12} \mathrm{H}_{12} \mathrm{~N}_{2} \mathrm{O}_{2}[\mathrm{M}]^{+} \cdot 216$, found 216 .

\subsubsection{N-(2-Oxo-4-(trifluoromethyl)-1,2-dihydroquinolin-7-yl)acetamide (10b)}

The title compound (10b) was obtained as brown crystals in $90 \%$ yield by following the general procedure. Melting point: > $300{ }^{\circ} \mathrm{C} ;{ }^{1} \mathrm{H}$ NMR (DMSO-d $6,300 \mathrm{MHz}$ ): $\delta 2.09$ (s, 3H, H-2) 6.79 (s, $\left.1 \mathrm{H}, \mathrm{H}-3\right), 7.35(\mathrm{~d}, 1 \mathrm{H}, \mathrm{J}=9.0 \mathrm{~Hz}, \mathrm{H}-6), 7.62(\mathrm{~d}, 1 \mathrm{H}$, $\mathrm{J}=8.7 \mathrm{~Hz}, \mathrm{H}-5), 7.98(\mathrm{~s}, 1 \mathrm{H}, \mathrm{H}-8) ; 13 \mathrm{C}$ NMR (DMSO-d, $75.5 \mathrm{MHz}): \delta 24.16(\mathrm{C}-2)$ ), 104.71, 108.61 (C-6 and C-8), 114.62 (C-3), 119.16 (C-10), 120.76 (C-5), 124.84 (C-9), $136.11-136.93$ (-CF $), 140.86$ (C-4), 142.06 (C-7), 160.41 (C-2), 169.12 (C-1'); HRMS: Calculated for $\mathrm{C}_{12} \mathrm{H}_{9} \mathrm{~F}_{3} \mathrm{~N}_{2} \mathrm{O}_{2}[\mathrm{M}+\mathrm{Na}]^{+} 293.0508$, found 293.0508. 


\subsection{CONCLUSION}

${ }^{1} \mathrm{H}$ NMR analysis of different derivatives of quinolones / coumarins led to the establishment of the following discriminative tools for the $\delta$ value for $\mathrm{H}-3$ :

1. It was observed that when C-4 position gets substituted with trifluoromethyl group, the proton attached with C-3 becomes highly downfield as compared to other substituents (methyl / hydroxy / acetoxy / amino).

2. The hydroxy group on the benzenoid ring causes shielding when incorporated in place of methoxy group and reverse trend was observed in case of acetoxy group as substituent.

3. In case of trifluoromethyl quinolone / coumarin derivatives, the maximum deshielding was observed in case of acetoxy derivative, whereby the acetoxy group was placed at C-6 position of benzenoid ring with $\Delta \delta$ of around 1.55 ppm.

\subsection{ACKNOWLEDGEMENT}

The financial assistance from Science and Engineering Research Board (DST), New Delhi is gratefully acknowledged.

\section{REFERENCES}

[1] N. Noda, Y. Yashiki, T. Nakatani, K. Miyahara, X.-M. Du. Chem. Pharm. Bull. 49 (2001) 930-931.

[2] I. Jacquemond-Collet, S. Hannedouche, I. Fourasté, C. Moulis. Fitoterapia 71 (2000) 605-606.

[3] N. F. de Moura, A. F. Morel, E. C. Dessoy, N. Zanatta, M. M. Burger, N. Ahlert, G. P. Porto, B. Baldisserotto. Planta Med. 68 (2002) 534-538.

[4] A. Lacy, R. O’ Kennedy. Curr. Pharm. Des. 10 (2004) 3797-3811.

[5] A. Kumar, B. K. Singh, R. Tyagi, S. K. Jain, S. K. Sharma, A. K. Prasad, H. G. Raj, R. C. Rastogi, A. C. Watterson, V. S. Parmar. Bioorg. Med. Chem. 13 (2005) 4300-4305.

[6] H. G. Raj, V. S. Parmar, S. C. Jain, E. Kohli, N. Ahmad, S. Goel, Y. K. Tyagi, S. K. Sharma, J. Wengel, C. E. Olsen. Bioorg. Med. Chem. 8 (2000) 1707-1712.

[7] A. Kathuria, A. Gupta, N. Priya, P. Singh, H. G. Raj, A. K. Prasad, V. S. Parmar, S. K. Sharma. Bioorg. Med. Chem. 17 (2009) 1550-1556.

[8] N. Priya, A. Gupta, K. Chand, P. Singh, A. Kathuria, H. G. Raj, V. S. Parmar, S. K. Sharma. Bioorg. Med. Chem. 18 (2010) 4085-4094.

[9] A. Kathuria, N. Priya, K. Chand, P. Singh, A. Gupta, S. Jalal, S. Gupta, H. G. Raj, V. S. Parmar, S. K. Sharma. Bioorganic and Medicinal Chemistry 20 (2012) 1624-1638.

[10] A. Maiti, P. V. N. Reddy, M. Sturdy, L. Marler, S. D. Pegan, A. D. Mesecar, J. M. Pezzuto, M. Cushman. J. Med. Chem. 52 (2009) 1873-1884.

[11] I. V. Ukrainets, L. V. Sidorenko, O. V. Gorokhova, N. A. Jaradat. Chem. Het. Comp. 42 (2006) 475-487.

[12] A. van Oeveren, M. Motamedi, N. S. Mani, K. B. Marschke, F. J. López, W. T. Schrader, A. Negro-Vilar, L. Zhi. J. Med. Chem. 49 (2006) 6143-6146.

[13] A. Rajendran, C. Karthikeyan, K. Rajathi, D. Ragupathy. J. Chem. Sci. 124 (2012) 877-881.

[14] M. Marull, O. Lefebvre, M. Schlosser. Eur. J. Org. Chem. 1 (2004) 54-63.

[15] W.M.F. Fabian, K.S. Niederreiter, G. Uray, W. Stadlbauer. J. Mol. Str. 477 (1999) 209-220.

[16] M. Kidwai, V. Bansal. Lett. Org. Chem. 4 (2007) 519.

[17] A.M. Nadzan, Jr. K.L. Rinehart. J. Am. Chem. Soc. 99 (1977) 4647-4654.

[18] G.R. Pettit, D.M. Piatak. J. Org. Chem. 25 (1960) 721.

[19] V.F. Traven, N.Y. Podhaluzina, A.V. Vasilyev, A.V. Manaev. ARKIVOC (2000) 931.

[20] R.R. Holmes, J. Conrady, J. Guthrie, R. McKay. J. Am. Chem. Soc. 76 (1954) 2400.

[21] H.A. Shoeb, M.I. Korkor, G.H. Tammam, S.M. El-Amin. Can. J. Pharm.Sci. 15 (1980) 66. 